\title{
Buttiauxella agrestis
}

National Cancer Institute

\section{Source}

National Cancer Institute. Buttiauxella agrestis. NCI Thesaurus. Code C86229.

A species of facultatively anaerobic, Gram-negative, bacilli assigned to the phylum

Proteobacteria. This species is motile using a peritrichous flagella, non-spore forming, oxidase negative, catalase positive, ferments sugar, reduces nitrate, and hydrolyzes esculin. B. agrestis is commensal in the throat and pathogenic in immunocompromised individuals with wound infections or appendicitis. 